\section{G204(P) MODELLING HIGH DEPENDENCY IN THE LOCAL NEONATAL UNIT}

1,2SJ Dalton, 'S Chahed, 'TJ Chaussalet. 'Computer Science, University of Westminster, London, UK; ${ }^{2}$ Paediatrics, Colchester General Hospital, Colchester, UK

10.1136/archdischild-2018-rcpch.199

Aim To evaluate service in an English local neonatal unit in regard of capacity issues with high dependency, which represents a lynchpin resource within the Perinatal Network System.

Methods. The research was performed in collaboration with a District General Hospital and a University Department of Computer Science. With the consent of the regional Research Ethics Committee, Standardised Electronic Neonatal Database (SEND) data cleaned by local neonatal unit ward clerks was analysed and patient pathways through high dependency modelled.

Results The unit configuration was one intensive care and four high dependency cots. During 2009-11, high dependency idle time was 0.14 , whilst oversubscription was 0.07 . $90 \%$ of daily changes in occupancy for high dependency were by up to one cot. The mean Length of Stay (LOS) was 5.5 days. For those patients requiring high dependency care after first being admitted to a different level of care, the mean prior stay on intensive care was 4.7 days and that on special care 2.9 days. In the population needing any high dependency, it represented a backward step in their care pathway in $4.7 \%$.

Diagnostic patient groups have differing LOS profiles. Those receiving high dependency for Neonatal Abstinence Syndrome (NAS) or Continuous Positive Airways Pressure for Chronic Lung Disease not only had the longest stays but also iterated between special care and high dependency. NAS accounted for $20 \%$ of high dependency work.

To achieve a less than even chance of overload in high dependency, spare capacity of two cots is needed. Having one unoccupied cot gives a $70 \%$ probability of overload. Up to 63 days per year are normally expected to be short of one or more nurses, assuming full nursing establishment and no absence.

Conclusion High dependency cot activity lurched between extremes. Economies of scale are difficult in medium sized local neonatal units. For a patient network, Game Theory tells us that load balancing of the whole system means running different occupancies in each component unit. We hope that this work contributes to understanding of patient networks and guides operations management, which is challenged by rota gaps.

\section{G205(P) FEASIBILITY STUDY OF A NOVEL ASSAY FOR DETECTION OF BACTERIA IN NEONATAL CSF}

${ }^{1} \mathrm{~A}$ Abelian, ${ }^{2} \mathrm{~T}$ Mund, ${ }^{3} \mathrm{M}$ Curran, ${ }^{4} \mathrm{~N}$ Mitra, ${ }^{4} \mathrm{C}$ Charan, ${ }^{4} \mathrm{~A}$ Ogilvy-Stuart, ${ }^{2} \mathrm{H}$ Pelham, ${ }^{5} \mathrm{PH}$ Dear. 'Paediatrics, Maelor Hospital, Wrexham, UK; ${ }^{2}$ Laboratory of Molecular Biology, Medical Research Council, Cambridge, UK; ${ }^{3}$ Clinical Microbiology, Addenbrookes Hospital, Cambridge, UK; ${ }^{4}$ Neonatal Unit, Rosie Maternity Hospital, Cambridge, UK; ${ }^{5}$ Mote Research Ltd, Cambridge, UK

\subsection{6/archdischild-2018-rcpch.200}

Aims An assay based on 16S rDNA PCR technology has been designed to detect a single intact bacterium whilst eliminating free DNA from dead bacteria, thus offering unprecedented sensitivity and scope to the analysis of bacterial carriage in clinical specimens. We hypothesised that application of such an assay to neonatal CSF will enable accurate, fast and inexpensive discrimination of bacteria-free specimens, and will have a small but clinically acceptable false-positive rate.

Methods Design of PCRctic - a novel assay based on 16S rDNA PCR technology utilising ethidium azide for elimination of free bacterial DNA and optimised for neonatal CSF - was presented at this conference in 2016. In this prospective study lasting 12 months, the feasibility of PCRctic was investigated in CSF specimens obtained from newborn babies tested for meningitis. Following interim analysis, sterile snap-top tubes (Eppendorf $^{\mathrm{TM}}$ ) replaced standard universal containers for collection of CSF, and ChloraPrep ${ }^{\mathrm{TM}}$ replaced Uniseptas the choice of antiseptic. Study received National REC and HRA approvals and was funded by the MRC.

Results Fifty-two specimens of CSF were tested before the interim analysis (1 st phase) and 21 after (2nd phase). In phase 1 , the assay detected bacteria in 19 specimens (36\%) and sequencing revealed several organisms of Flavobacteriaceae family (Cloacibacterium, Flavobacterium, Hymenobacter), as well as Ochrobactrum (Brucellaceae), Sneathia amnii (Leptotrichiaceae), Pseudomonas spp, Acinetobacter, Sphingomonadaceae, Oscillatoriales (Cyanobacteria), Ureaplasma urealyticum, Staphylococcus auricularis, Streptococcus spp, Bdellovibrio, Aerococcus christensenii, Methylobacterium, and Pedobacter (Sphingomonadaceae). In phase 2, bacteria were detected in two specimens $(9.5 \%)$ and sequencing revealed Geobacter in one and mixed spp in the other. No clinical cases of neonatal bacterial meningitis occurred during the study. A positive signal was detected in only one out of 23 negative controls designed to test for environmental contamination (4\%), sequencing revealed Bacillus.

Conclusion The assay's rate of positive results decreased significantly following simple steps to reduce the risk of contamination at the time of CSF collection. Using additional inexpensive measures it may be possible to reduce the rate further and begin to explore the introduction of the assay into practice.

\section{G206(P) GROWTH AND HEALTHCARE UTILISATION IN PREMATURE BABIES DISCHARGED ON HOME OXYGEN}

R Pang, H Williams-Gunn, A Sinha, P Rughani, M Hird, C May. Neonatology, Royal London Hospital, Barts Health NHS Trust, London, UK

\subsection{6/archdischild-2018-rcpch.201}

Background Premature infants with bronchopulmonary dysplasia (BPD) are a vulnerable group of babies. Home oxygen provision facilitates early discharge, with the aim of minimising disruption to family life, hopefully optimising growth and reducing the financial burden on the NHS. We carried out a study to evaluate the growth and healthcare utilisation after discharge.

Method Data was collected retrospectively from electronic patient records for infants born between 2011-2014, discharged on home oxygen and followed up in clinic until 2 years old. This was evaluated against a comparison group who did not need home oxygen, matched for gestation. Weight SD scores were calculated using LMS calculator for Microsoft Excel. Data was analysed using SPSS.

Results Thirty-six babies discharged with home oxygen were evaluated against a comparison group of 19 babies born 